Research Article

\title{
Exploring the Mechanism of Baicalin Intervention in Breast Cancer Based on MicroRNA Microarrays and Bioinformatics Strategies
}

\author{
Anqi Ge $\mathbb{D}^{1},{ }^{1}$ Lifang Liu $\mathbb{D}^{1},{ }^{1}$ Xian'guang Deng, ${ }^{1}$ Jun Luo, ${ }^{1,2}$ and Yanghua $X u^{2}$ \\ ${ }^{1}$ The First Affiliated Hospital of Hunan University of Chinese Medicine, Changsha, Hunan, China \\ ${ }^{2}$ Hunan University of Chinese Medicine, Changsha, Hunan, China \\ Correspondence should be addressed to Anqi Ge; geanqi32121@163.com and Lifang Liu; liulff@126.com
}

Received 7 May 2021; Accepted 2 November 2021; Published 20 December 2021

Academic Editor: Sri Fatmawati

Copyright (C) 2021 Anqi Ge et al. This is an open access article distributed under the Creative Commons Attribution License, which permits unrestricted use, distribution, and reproduction in any medium, provided the original work is properly cited.

\begin{abstract}
Objective. To explore the mechanism of baicalin intervention in breast cancer based on microRNA microarrays. Methods. The inhibitory rate of baicalin intervention in MCF-7 breast cancer cells was determined by MTT. Then, the miRNA microarrays were used to validate the key microRNAs. After that, reverse transcription-quantitative polymerase chain reaction (RT-qPCR) was used to validate microRNA, hsa-miR-15a, hsa-miR-100, hsa-miR-16, and hsa-miR-7t. Finally, the potential targets of these key microRNAs are predicted by miRWalk, and DAVID was utilized for gene ontology (GO) enrichment analysis and pathway enrichment analysis. Results. Baicalin may inhibit the proliferation of MCF-7 cells in a dose-dependent and time-dependent manner. The concentration of baicalin $150 \mu \mathrm{mol} / \mathrm{L}$ was determined for the subsequent miRNA chip research. A total of 92 upregulated microRNAs and 35 downregulated microRNAs were obtained. The upregulated miRNAs include hsa-miR-6799-5p, hsa-miR-6126, hsa-miR-4792, hsa-miR-6848-5p, hsa-miR-3197, hsa-miR-6779-5p, and hsa-miR -654-5p. The downregulated miRNAs include hsa-miR-3911, hsa-miR-504-5p, hsa-miR-30a-3p, hsa-miR-193b-3p, and hsa-miR-181b-5p. Then, differentially expressed miRNA was verified by qRT-PCR. The results showed that the expression of hsa-miR-15a, hsa-miR-100, hsa-miR-16, and hsa-let-7c was upregulated $(P<0.05)$, which was consistent with the results of the miRNA microarray. The enrichment analysis showed that baicalin might regulate the DNA-templated proliferation, DNA-templated transcription, p53 signaling pathway, etc., of MCF-7 breast cancer cells through miRNA. Conclusion. Baicalin inhibits the proliferation of breast cancer cells. It may achieve antitumor effects through regulating microRNAs so as to affect the DNA replication (such as cellular response to DNA damage stimulus and DNA binding), RNA transcription (such as regulation of transcription, DNA-templated, transcription from RNA polymerase II promoter, and transcription factor binding), protein synthesis (such as mRNA binding, Golgi apparatus, and protein complex), endocytosis, pathways in cancer, p53 signaling pathway, and so on.
\end{abstract}

\section{Introduction}

As the global incidence of cancer rises sharply, breast cancer is one of the most common malignant tumors among women and has become the leading cause of cancer death in women. Worldwide, there are approximately 2.1 million newly diagnosed female breast cancer cases in 2018, accounting for a quarter of female cancers $[1,2]$. Female breast cancer is the most common type of cancer in most countries $(154 / 185)$ and is the leading cause of cancer deaths in more than 100 countries [3]. Breast cancer might result from multiple factors such as reproductive related factors, family accumulation and genetic factors, dietary factors, and breast disease history [4]. Breast cancer could be divided into four molecular subtypes based on molecular markers: luminal A type, luminal B type, triple-negative type, and HER2overexpressive type $[5,6]$.

At present, the treatment of breast cancer is mainly based on surgical treatment and radiotherapy and chemotherapy, supplemented by targeted therapy and biological therapy. In adjuvant therapy, patients with luminal $\mathrm{A}$ - and luminal B-type breast cancer are mainly treated with endocrine 
therapy, and chemotherapy is mainly performed in patients with triple-negative breast cancer. In patients with HER2overexpressing breast cancer, targeted therapy is used to target the HER2 gene $[5,6]$. However, due to the toxic side effects and drug resistance of radiotherapy and chemotherapy, patients with tumor survival often have poor prognosis, making breast cancer treatment effect not ideal [7-9]. Therefore, finding and developing sensitizing drugs and antitumor drugs with good safety and low side effects have become the key to cancer treatment.

Baicalin is a flavonoid extracted from the dried roots of Scutellaria baicalensis Georgi. Current studies show that baicalin inhibits various biological behaviors of breast cancer, such as apoptosis, autophagy, epithelial-mesenchymal transition, migration, invasion, and metastasis, and enhances the sensitization of chemotherapy [10]. Its specific mechanism is regulation of the NF- $\kappa \mathrm{B}$ signaling pathway [11], Wnt/ $\beta$-catenin signaling pathway [12], transforming growth factor (TGF)- $\beta$ signaling pathway [13], AMPK/ ULK1/mTOR signaling pathway [14], and ERK/p38MAPK signaling pathway [15]. However, the regulation mechanism of baicalin on breast cancer bionetworks still requires new strategies to discover. More importantly, recent studies have demonstrated that microRNAs play an important role in breast cancer bionetworks that regulate breast cancer proliferation, metastasis, and invasion by targeting downstream signaling pathways [16-18]. Therefore, this study integrated the microRNA microarrays and bioinformatics to discover the regulation mechanism of baicalin on breast cancer bionetworks. We also hope to provide a new paradigm for future research by designing a new strategy.

\section{Materials and Methods}

2.1. Experimental Drugs. Baicalin was purchased from China National Institute for the Control of Pharmaceutical and Biological Products, with a purity of $98.5 \%$ and product batch no. 120608-201113. The baicalin dry powder was fully dissolved in dimethyl sulfoxide (DMSO) and formulated into a stock solution with a concentration of $5000 \mu \mathrm{mol} / \mathrm{L}$, protected from light and stored at $4^{\circ} \mathrm{C}$. The final concentration was $0 \mu \mathrm{mol} / \mathrm{L}(\mathrm{NC}), 50 \mu \mathrm{mol} / \mathrm{L}, 100 \mu \mathrm{mol} / \mathrm{L}$, and $150 \mu \mathrm{mol} / \mathrm{L}$, diluted with the corresponding medium and DMSO.

\subsection{Cell Lines of Breast Cancer and Normal Breast Cells.} Human breast cancer cell line MCF-7 cells were purchased from the Cell Center of Xiangya School of Medicine, Central South University. Normal breast Hs 578Bst cell line was purchased from Shanghai Baili Biotechnology Co., Ltd. All were prepared by American Standard Biological Products Collection (ATCC).

The breast cancer cell line was cultured in the RPMI 1640 medium (containing 10\% calf serum, penicillin $100 \mathrm{U} / \mathrm{ml}$, and streptomycin $100 \mathrm{U} / \mathrm{ml}$ ), cultured at $37^{\circ} \mathrm{C}, 100 \% \mathrm{hu}-$ midity, $5 \%$ (volume fraction) $\mathrm{CO}_{2}$ incubator. Change the medium every 3 days. After the cells grow adherently, $0.25 \%$ trypsin is used for digestion and passage. Cells in the logarithmic growth phase were used for further experiments.

2.3. Reagents and Instruments. Penicillin/streptomycin $(100 \mathrm{x})$ and fetal bovine serum (FBS) were purchased from Sciencell Inc. D-Hank's solution, trypsin, and PBS buffer were obtained from the Central Laboratory of Xiangya Medical College. $0.05 \%$ trypsin $+0.38 \mathrm{mM}$ EDTA was purchased from Gibco Inc. Cell culture plates were purchased from Costar Inc. Cell cryopreservation tube was purchased from China Biyuntian Biotechnology Research Institute. Agarose and DMSO were purchased from Gaylord Slidell Inc. Whole Transcriptome Amplification Kit (Real Time) Ver. 2 and real-time quantitative RT- PCR detection (qRTPCR) kit were purchased from Bao Bioengineering (Dalian) Co., Ltd.; GoldVew nucleic acid dye was purchased from Shanghai Shenggong Bioengineering Technology Service Co., Ltd. Lipofectamine 2000 transfection reagent was purchased from Invitrogen Inc. Plasmid Purification Kit was purchased from QIAGEN Inc. Ordinary plasmid small kit was purchased from Shanghai Shenggong Bioengineering Technology Service Co., Ltd. MicroRNA microarrays were purchased from Guangzhou Ruibo Biotechnology Co., Ltd. Total RNA extraction reagent TRIzol Reagent was purchased from Invitrogen Inc.; reverse transcription kit-SYBR Premix Ex TaqTM II (DRR081A)-was purchased from Takara Inc. The primers are shown in Table 1.

2.4. Cell Proliferation Measured by the MTT Assay. MCF-7 cells that grew in the logarithmic phase with good condition were incubated in a $5 \% \mathrm{CO}_{2}, 37^{\circ} \mathrm{C}$ incubator. Then, baicalin at concentrations of $0 \mu \mathrm{mol} / \mathrm{L}$ (NC), $50 \mu \mathrm{mol} /$ $\mathrm{L}, 100 \mu \mathrm{mol} / \mathrm{L}$, and $150 \mu \mathrm{mol} / \mathrm{L}$ was added separately. After incubating in a $5 \% \mathrm{CO}_{2}, 37^{\circ} \mathrm{C}$ incubator for 24,48 , and 72 hours, the cells were observed under an inverted microscope. Then, the MTT solution was added to terminate the culture, and finally, DMSO was added to each well. The enzymelinked immunosorbent assay was used to measure the absorbance of each well at OD $490 \mathrm{~nm}$.

\subsection{Total RNA Extraction and miRNA Microarrays'Screening.} Total RNA of MCF-7 cells treated with an optimum concentration of baicalin (experiment group) and the MCF-7 cells not treated with baicalin (control group) was extracted by using TRIzol and miRNA extraction kits according to the manufacturer's instructions, which effectively covered all types of RNA, including miRNA. The nanodrop spectrophotometer was used to measure the quality and quantity of RNA, and the integrity of RNA was measured by gel electrophoresis. miRNA was labeled with the T4RNA ligase labeling method and precipitated with absolute ethanol, and then miRNA microarray hybridization scanning and data analysis were completed. Differential expression genes of breast cancer cells after baicalin intervention were screened by the chip saliency analysis algorithm (SAM 3.11). 
Table 1: Primers.

\begin{tabular}{lrc}
\hline miRNA & Forward primer & Reverse primer \\
\hline miR-15a & $5^{\prime}$-GGGTAGCAGCACATAATGG-3' \\
miR-100 & $5^{\prime}$-GCTCTGAACCGTAGATCCGAAC-3' \\
miR-16 & $5^{\prime}$-GGGTAGCAGCACGTAAATA-3' & $5^{\prime}$-CAGTGCGTGTCGTGGAGT-3' \\
miR-let-7c & $5^{\prime}$-GCCGCTGAGGTAGTAGGTTGTAT-3 ${ }^{\prime}$ & $5^{\prime}$-GTGCAGGGTCCGAGGT-3' \\
U6 & $5^{\prime}$-CTCGCTTCGGCAGCACATATACT-3' & $5^{\prime}$-CAGTGCGTGTCGTGGAGT-3' \\
\hline
\end{tabular}

2.6. Validation of miRNA Expression through Real-Time Fluorescence Quantification of PCR ( $q R T-P C R)$. Total RNA of the blank group and the baicalin group was $500 \mathrm{ng}$. RNA was mixed with $2 \mu \mathrm{ldNTP}(2.5 \mathrm{mmol} / \mathrm{L}), 2 \mu \mathrm{l} 10 \mathrm{X}$ RT buffer, $0.3 \mu \mathrm{l}$ RT-specific primer $(1 \mu \mathrm{mol} / \mathrm{L}), 0.2 \mu \mathrm{l}$ MMLV reverse transcriptase $(200 \mathrm{U} / \mu \mathrm{l}), 0.3 \mu \mathrm{l}$ RNase inhibitor $(40 \mathrm{U} / \mu \mathrm{l})$, and $20 \mu \mathrm{l}$ RNA enzyme-free water. cDNA was synthesized in the PCR thermal cycler. The data were analyzed by the $2^{-\Delta \Delta C T}$ method. The experiment was repeated 3 times under the same conditions.

2.7. Potential Targeted Genes of MicroRNA Prediction and Enrichment Analysis. The potential targeted genes of miRNA were predicted by miRWalk 3.0 (http://mirwalk. umm.uni-heidelberg.de/) $[19,20]$. The DAVID database ver. 6.8 (https://david-d.ncifcrf.gov) was applied for gene ontology (GO) enrichment analysis and pathway enrichment analysis [21].

2.8. Statistical Analysis. The data were expressed as $x \pm s$, and SPSS 22.0 statistical software was used for processing, and the $t$-test was used for comparison of differences between groups. $P<0.05$ means that the difference was statistically significant.

\section{Results}

3.1. Inhibition of Baicalin on the Proliferation of Breast Cancer Cell MCF-7. Baicalin at concentrations of 0,50, 100, 150, and $200 \mu \mathrm{mol} / \mathrm{L}$ was added and interfered with breast cancer cell MCF-7 for 24, 48, and 72 hours. The growth of breast cancer cell MCF-7 was significantly inhibited and was time and concentration dependent within a certain time and concentration range. The differences between the baicalin groups and the negative control group were statistically significant $(P<0.05)$ (Figure 1$)$.

3.2. Differentially Expressed MicroRNA. The concentration of baicalin $150 \mu \mathrm{mol} / \mathrm{L}$ was determined for the subsequent miRNA chip research. $\log 2 \mathrm{FC} \geq 1$ or $\leq-1$ and $P<0.05$ were used as the standard to screen for differentially expressed microRNA. Finally, a total of 92 upregulated microRNAs and 35 downregulated microRNAs were obtained (Figure 2). The most upregulated miRNAs include hsa-miR-6799-5p, hsa-miR-6126, hsa-miR-4792, hsa-miR-6848-5p, hsa-miR3197, hsa-miR-6779-5p, and hsa-miR -654-5p. The most downregulated miRNAs include hsa-miR-3911, hsa-miR504-5p, hsa-miR-30a-3p, hsa-miR-193b-3p, and hsa-miR$181 \mathrm{~b}-5 \mathrm{p}$.

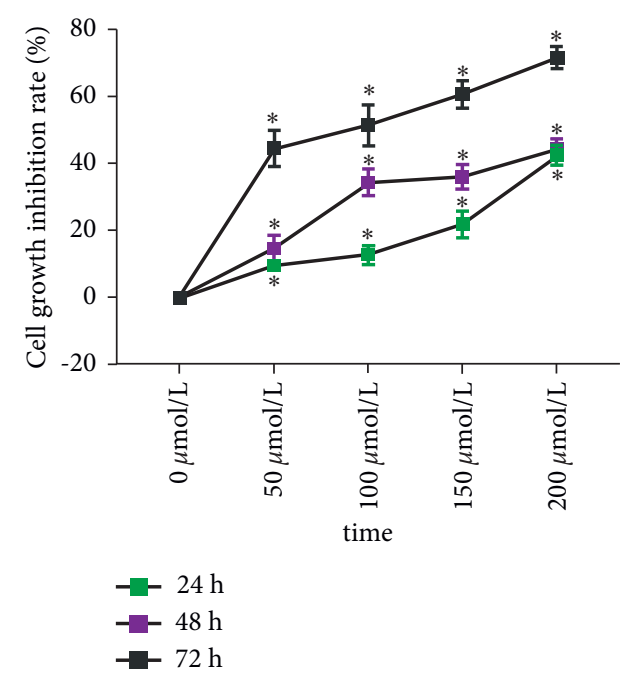

Figure 1: Inhibition of baicalin on the proliferation of breast cancer cell MCF-7 (* compared with the control group, $P<0.05$ ).

3.3. Bioinformatics Analysis of Differentially Expressed MicroRNA. miRWalk was utilized to obtain potential mRNA of differentially expressed microRNA. Potential mRNAs were input into DAVID for enrichment analysis (Table S1). The biological processes include transcription, DNA-templated, regulation of transcription, DNA-templated, negative regulation of transcription from RNA polymerase II promoter, positive regulation of transcription, DNA-templated, negative regulation of transcription, DNA-templated, cellular response to hypoxia, protein autophosphorylation, cell-cell adhesion, transcription from RNA polymerase II promoter, cellular response to DNA damage stimulus, and protein ubiquitination (Figure 3(a)). The cell components include the nucleoplasm, nucleus, cytosol, membrane, cytoplasm, nucleolus, endoplasmic reticulum membrane, nuclear chromatin, cell-cell adherens junction, intracellular, nuclear body, Golgi apparatus, protein complex, focal adhesion, and clathrin-coated pit (Figure 3(b)). The molecular functions include protein binding, poly(A) RNA binding, DNA binding, ubiquitin-protein ligase binding, nucleic acid binding, transcription factor binding, metal ion binding, transcription factor activity, sequence-specific DNA binding, GDP binding, RNA polymerase II core promoter proximal region sequence-specific DNA binding, mRNA binding, and ubiquitin-protein transferase activity (Figure 3(c)). The signaling pathways include pancreatic cancer, endocytosis, pathways in cancer, p53 signaling pathway, prostate cancer, glioma, colorectal cancer, Hippo signaling pathway, FoxO signaling pathway, chronic myeloid leukemia, and signaling pathways regulating pluripotency of stem cells (Figure 3(d)). 


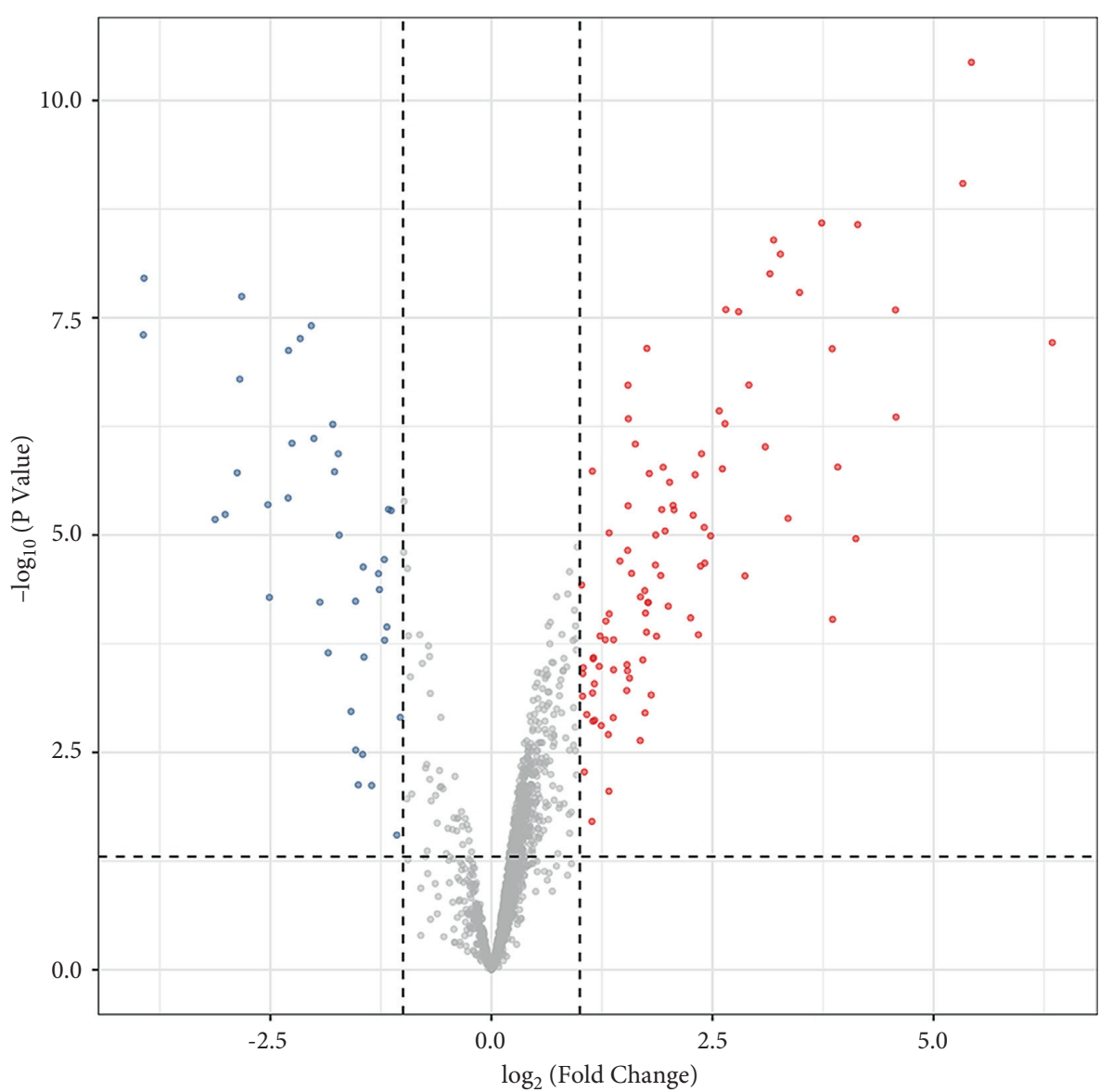

Group

- Down-regulated

- not-significant

- Up-regulated

(a)

FIgURE 2: Continued. 


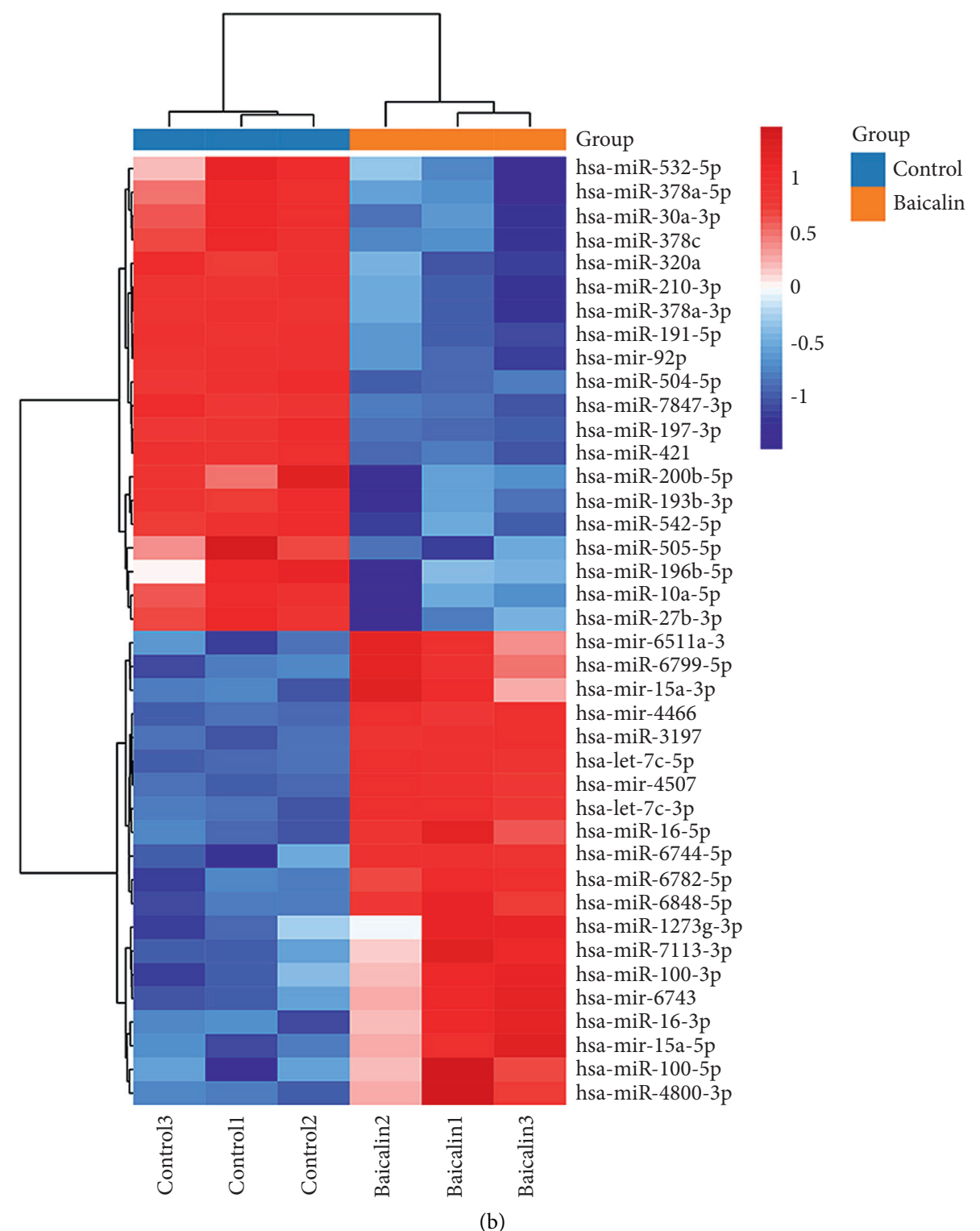

FIGURE 2: Differentially expressed microRNAs. (a) Volcano map. (b) Clustering heat map.

3.4. Validation of hsa-miR-15a, hsa-miR-100, hsa-miR-16, and hsa-let-7c in the Microarrays' Results Using $q R T-P C R$. The PCR electropherogram showed that the internal reference and miRNA bands were clearly visible, indicating that the extracted total RNA was relatively intact and substantially free of degradation. The UV spectrophotometer showed that the absorbance values of the samples at 260-280 $\mathrm{nm}$ were between 1.8 and 2.0, indicating that the purity meets the requirements. Then, qPCR was utilized to detect the expression of hsa-miR-15a, hsa-miR-100, hsamiR-16, and hsa-let-7c miRNAs. The results showed that the expression of hsa-miR-15a, hsa-miR-100, hsa-miR-16, and hsa-let-7c was upregulated $(P<0.05)$. This was consistent with the results of the miRNA microarray (Figure 4 ).

\section{Discussion}

MicroRNA (or miRNA) is a small noncoding RNA molecule ranging in length from 20 to 25 nucleotides that binds primarily to the $3^{\prime}$ untranslated region (UTR) of messenger RNA, resulting in downregulation of the target protein by degradation or translational inhibition of mRNA [22]. miRNAs are not directly encoded by their corresponding genes. RNA polymerase II in the nucleus can transcribe miRNA genes to form PRI-miRNAs. Next, PRI-miRNA is further cleaved by a complex of Drosha enzyme and Pasha/ DGCR8 protein into precursor miRNA (pre-miRNA) containing approximately 70 nucleotides [23]. The transporter Exportin-5 cleaves pre-miRNA into a miRNA duplex 


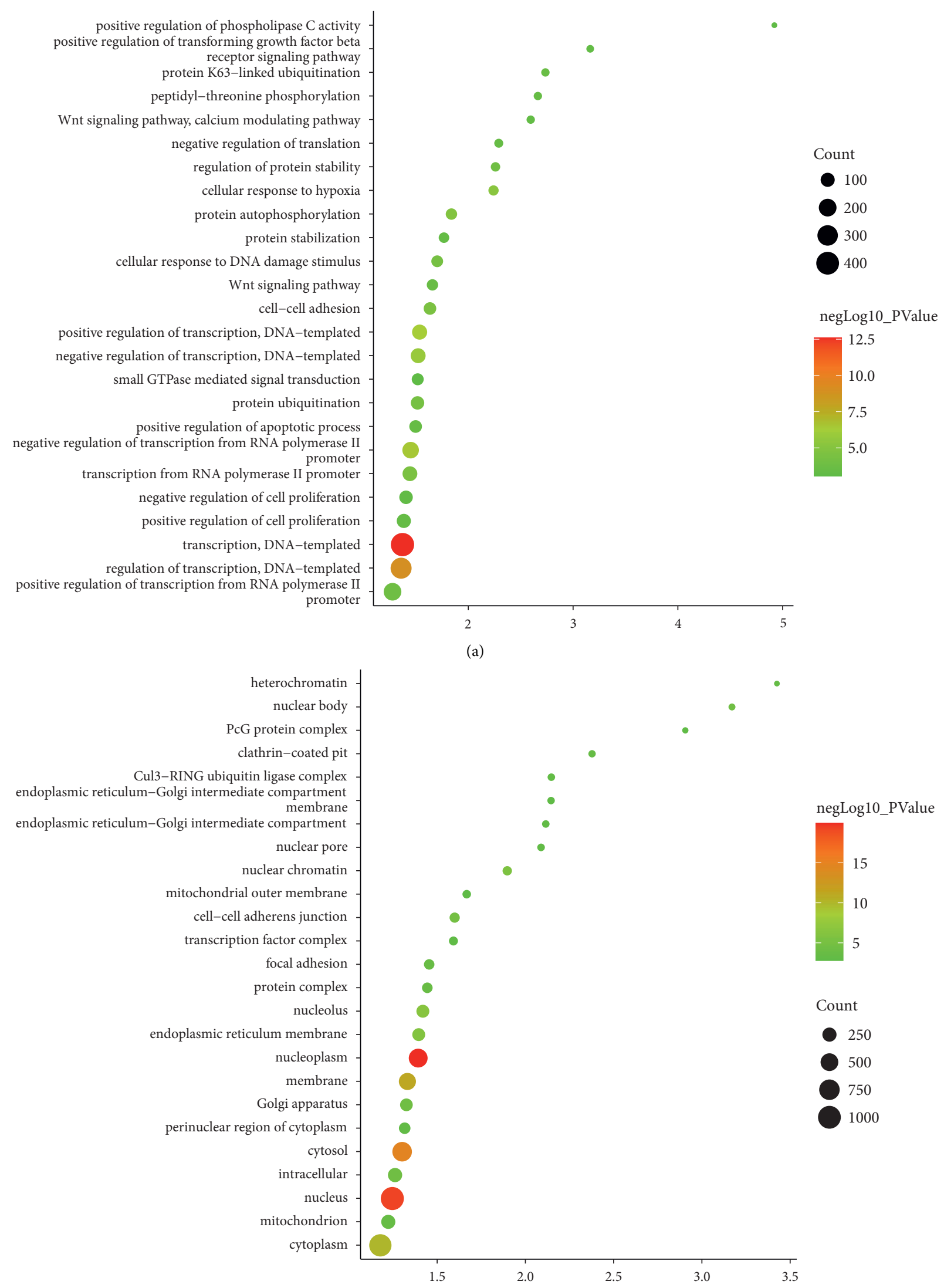

(b)

FIGURE 3: Continued. 


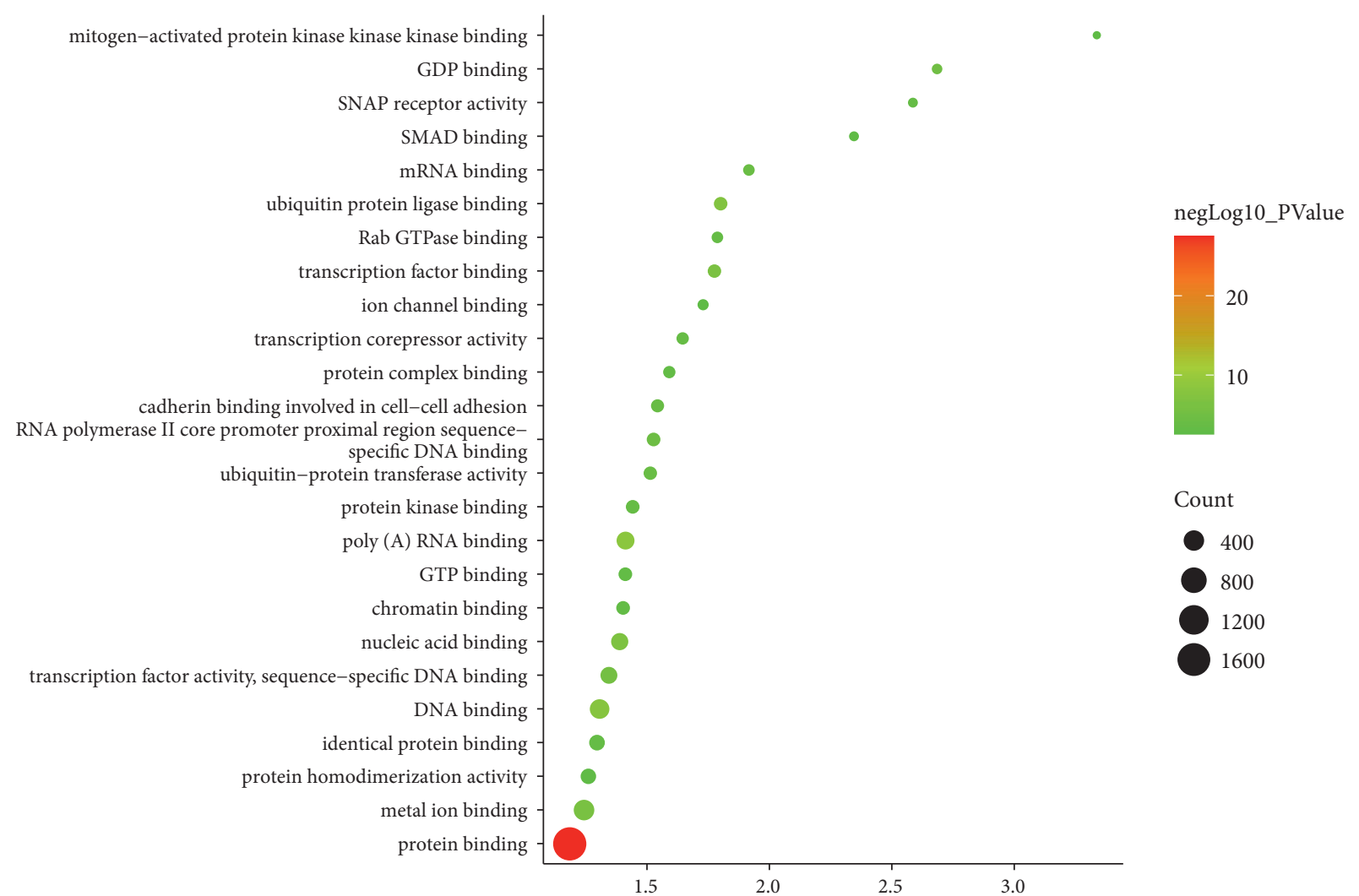

(c)

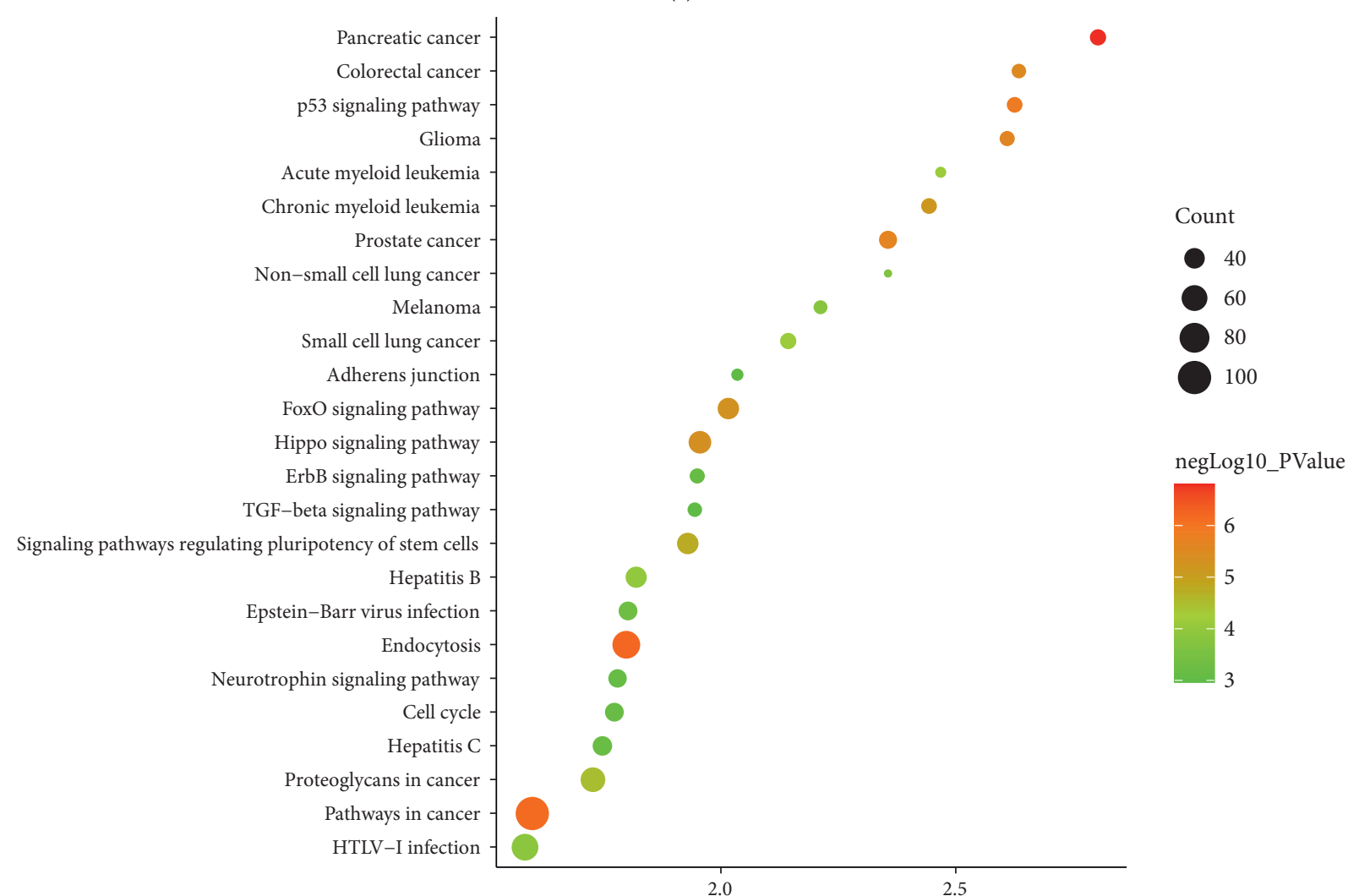

(d)

FiguRe 3: Bioinformatics analysis of differentially expressed microRNAs. (a) Biological processes. (b) Cell components. (c) Molecular functions. (d) Signaling pathways. $X$-axis represents fold enrichment. 


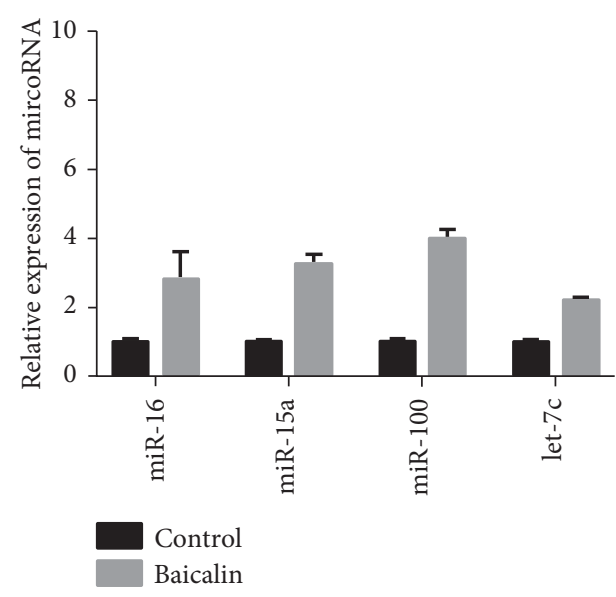

FIGURE 4: Validation of hsa-miR-15a, hsa-miR-100, hsa-miR-16, and hsa-let-7c in the microarrays' results using qRT-PCR.

containing approximately 22 nucleotides, with the mature single-stranded miRNA retained and the other singlestranded RNA degraded [24, 25]. The total number of miRNAs in human genes is expected to exceed one thousand and regulate approximately $30 \%$ of human genes. In vivo, miRNA regulates the expression of its target genes mainly by inducing degradation of target mRNA and inhibiting translation of target mRNA [26]. In breast cancer cells, miRNAs can bind to the mRNA $3^{\prime}$-UTR of a specific gene through complementary pairing, resulting in inhibition of translation of the target gene, thereby regulating gene expression and function. As an endogenous inhibitor of gene expression, miRNA forms a complex intracellular network with its upstream and downstream effector molecules, regulating the process of cell life activities, such as the occurrence and development of tumor cells [27, 28].

Recent studies have shown that dysregulation of miRNAs not only affects cellular processes associated with carcinogenesis, tumor resistance, invasion, and metastasis but may also have a direct impact on the effectiveness of treatment [29, 30]. Among them, there are oncogenic miRNA and tumor suppressor miRNA, which act on tumor suppressor genes and proto-oncogenes, respectively. These miRNAs regulate the progression of breast cancer in drug metabolism enzymes, breast cancer stem cells, and singlenucleotide polymorphisms in breast cancer [31]. For example, compared with normal breast tissue, the expression of miR-21 and miR-155 was upregulated, and that of miR10b, miR-125b, and miR-145 was downregulated in breast cancer tissues [32]. Volinia et al. found that miR-17-5p, miR20a, miR-21, miR-106a, and miR-155 were closely related to tumorigenesis in tumor tissues such as breast cancer [33]. At present, the research found that proto-oncogene miRNAs closely related to breast cancer are miR-10b, miR-21, miR155 , miR-181a/181b, and so on [34], while tumor suppressor miRNAs include the lethal-7 family (Let-7), miR-125a/125b, miR-206, and miR-34 [35].

miRNAs also play an important role in the regulation of breast cancer stem cells. Shimono et al. showed a decrease in the expression of the miR-200 family (miR-200a/-200b/-200c,
miR-101, miR-182miR-183, and miR-96) in CD44+CD24-/low lineage-primary breast cancer stem cells [36]. miR-200c also strongly inhibits the formation of mammary ducts and the ability of breast cancer stem cells to form tumors in normal breast stem cells [33]. In the cancer cells of breast cancer with bone metastasis and lung metastasis, the expression of 8 miRNAs decreased, including miR-126, miR-206, and miR335; these miRNAs also decreased in distant metastatic cancer cells in other sites $[37,38]$. MicroRNAs also begin to play a role in early diagnosis and typing of breast cancer $[39,40]$, such as luminal type A (upregulated expression: let-7a/b/c/f, miR-10a, -30a-3p, -30a-5p, -99a, -100, -126, -130a, -136, -145, -199a/b, $-224,-214$, and -342) and luminal type $\mathrm{B}$ (upregulated expression: let-7b/c/f, miR-10a, -30a-3p, -30a-5p, -93, -25, -106a/ b, -224, and -342).

Recent studies indicate that miRNAs are key regulators of these drug transporters, such as miRNAs targeting MDR1 including miR-451 [41], miR-7, miR-345 [42], and miR-326 [43]. Increased expression of miRNAs associated with response to radiation therapy results in increased resistance of the tumor to treatment. Many miRNAs that have been found to be involved in drug resistance of breast cancer include the miRNA-let-7 family, miR-451, miR-21, miR-101, and miR$221 / 222[44,45]$. miRNAs associated with resistance to endocrine therapy include miR-221, -222 , and $-181 \mathrm{~b}$, miR30c, and miR-301 [46, 47].

In summary, miRNA dysregulation in breast cancer, whether through polymorphisms in miRNA sequences, binding sites in target genes, or epigenetic mechanisms, has been shown to play a key role in the entire carcinogenesis process. Meanwhile, this study shows that baicalin can interfere with these miRNAs, suggesting that baicalin may exert antitumor effects on breast cancer at miRNA levels.

\section{Conclusion}

Baicalin inhibits the proliferation of breast cancer cells. It may achieve antitumor effects through regulating microRNAs so as to affect the DNA replication (such as cellular response to DNA damage stimulus and DNA binding), RNA transcription (such as regulation of transcription, DNAtemplated, transcription from RNA polymerase II promoter, and transcription factor binding), protein synthesis (such as mRNA binding, Golgi apparatus, and protein complex), endocytosis, pathways in cancer, p53 signaling pathway, and so on.

\section{Data Availability}

The data that support the findings of this study are openly available in supplementary materials.

\section{Conflicts of Interest}

The authors declare no conflicts of interest.

\section{Authors' Contributions}

Anqi Ge, Yanghua $\mathrm{Xu}$, and Lifang Liu dominated the concept and carried out a comprehensive design. Anqi Ge, 
Yanghua $\mathrm{Xu}$, Xian'guang Deng, Jun Luo, and Lifang Liu were responsible for data analysis and interpretation. Anqi $\mathrm{Ge}$, Yanghua Xu, Xian'guang Deng, and Jun Luo drafted the paper. Lifang Liu supervised the study. All authors participated in the analysis and interpretation of data and approved the final paper.

\section{Acknowledgments}

This work was supported by the Scientific Research Fund of Hunan Provincial Education Department (no. 19B434) and the Natural Science Foundation of Hunan Province, China (no. 2021JJ40421).

\section{Supplementary Materials}

Table S1: bioinformatics analysis of differentially expressed microRNAs. (Supplementary Materials)

\section{References}

[1] B. Freddie, F. Jacques, S. Isabelle, R. L. Siegel, L. A. Torre, and J. Ahmedin, "Global cancer statistics 2018: GLOBOCAN estimates of incidence and mortality worldwide for 36 cancers in 185 countries," CA: A Cancer Journal for Clinicians, vol. 68, pp. 394-424, 2018.

[2] C. Allemani, T. Matsuda, V. Di Carlo et al., "Global surveillance of trends in cancer survival 2000-14 (CONCORD3): analysis of individual records for 37,513,025 patients diagnosed with one of 18 cancers from 322 population-based registries in 71 countries," Lancet, vol. 391, pp. 1023-1075, 2018.

[3] J. Ferlay, I. Soerjomataram, R. Dikshit et al., "Cancer incidence and mortality worldwide: sources, methods and major patterns in GLOBOCAN 2012," International Journal of Cancer, vol. 136, no. 5, pp. E359-E386, 2015.

[4] L. Del Pup, G. Codacci-Pisanelli, and F. Peccatori, "Breast cancer risk of hormonal contraception: counselling considering new evidence," Critical Reviews in Oncology/Hematology, vol. 137, pp. 123-130, 2019.

[5] Y. Tang, Y. Wang, M. F. Kiani, and B. Wang, "Classification, treatment strategy, and associated drug resistance in breast cancer," Clinical Breast Cancer, vol. 16, no. 5, pp. 335-343, 2016.

[6] X. Deng, H. Wu, F. Gao et al., "Brachytherapy in the treatment of breast cancer," International Journal of Clinical Oncology, vol. 22, no. 4, pp. 641-650, 2017.

[7] Y.-Z. Jiang, K.-D. Yu, W.-T. Peng et al., "Enriched variations in TEKT4 and breast cancer resistance to paclitaxel," Nature Communications, vol. 5, no. 1, p. 3802, 2014.

[8] A. Britschgi, R. Andraos, H. Brinkhaus et al., "Abstract lb-36: jak2/stat5 inhibition circumvents resistance to pi3k/mtor blockade: a rationale for co-targeting these pathways in metastatic breast cancer," Cancer Cell, vol. 22, pp. 796-811, 2013.

[9] A. Ray Chaudhuri, E. Callen, X. Ding et al., "Replication fork stability confers chemoresistance in BRCA-deficient cells," Nature, vol. 535, no. 7612, pp. 382-387, 2016.

[10] C.-P. Yu, Y.-C. Hsieh, C.-S. Shia et al., "Increased systemic exposure of methotrexate by a polyphenol-rich herb via modulation on efflux transporters multidrug resistance-associated protein 2 and breast cancer resistance protein,"
Journal of Pharmaceutical Sciences, vol. 105, no. 1, pp. 343349, 2016.

[11] Y. Gao, H. Liu, H. Wang et al., "Baicalin inhibits breast cancer development via inhibiting ІкВ kinase activation in vitro and in vivo," International Journal of Oncology, vol. 53, pp. 2727-2736, 2018.

[12] T. Zhou, A. Zhang, G. Kuang et al., "Baicalin inhibits the metastasis of highly aggressive breast cancer cells by reversing epithelial-to-mesenchymal transition by targeting $\beta$-catenin signaling," Oncology Reports, vol. 38, pp. 3599-3607, 2017.

[13] H. Chung, H. S. Choi, E.-K. Seo, D.-H. Kang, and E.-S. Oh, "Baicalin and baicalein inhibit transforming growth factor$\beta 1$-mediated epithelial-mesenchymal transition in human breast epithelial cells," Biochemical and Biophysical Research Communications, vol. 458, no. 3, pp. 707-713, 2015.

[14] C. Yu, Z. Zhang, H. Zhang et al., "Pretreatment of baicalin and wogonoside with glycoside hydrolase: a promising approach to enhance anticancer potential," Oncology Reports, vol. 30, no. 5, pp. 2411-2418, 2013.

[15] Q.-m. Zhou, S. Wang, H. Zhang et al., "The combination of baicalin and baicalein enhances apoptosis via the ERK/p38 MAPK pathway in human breast cancer cells," Acta Pharmacologica Sinica, vol. 30, no. 12, pp. 1648-1658, 2009.

[16] C. Graveel, H. Calderone, J. Westerhuis, M. Winn, and L. Sempere, "Critical analysis of the potential for microrna biomarkers in breast cancer management," Breast Cancer: Targets and Therapy, vol. 59, 2015.

[17] A. Tahiri, M. R. Aure, and V. N. Kristensen, "MicroRNA networks in breast cancer cells," Methods in Molecular Biology, vol. 1711, pp. 55-81, 2018.

[18] M. Adhami, A. A. Haghdoost, B. Sadeghi, and R. Malekpour Afshar, "Candidate miRNAs in human breast cancer biomarkers: a systematic review," Breast Cancer, vol. 25, no. 2, pp. 198-205, 2018.

[19] M. D. Paraskevopoulou, G. Georgakilas, N. Kostoulas et al., "DIANA-microT web server v5.0: service integration into miRNA functional analysis workflows," Nucleic Acids Research, vol. 41, no. W1, pp. W169-W173, 2013.

[20] M. Reczko, M. Maragkakis, P. Alexiou, I. Grosse, and A. G. Hatzigeorgiou, "Functional microRNA targets in protein coding sequences," Bioinformatics, vol. 28, no. 6, pp. 771-776, 2012.

[21] D. W. Huang, B. T. Sherman, and R. A. Lempicki, "Systematic and integrative analysis of large gene lists using DAVID bioinformatics resources," Nature Protocols, vol. 4, no. 1, pp. 44-57, 2009.

[22] J. Winter, S. Jung, S. Keller, R. I. Gregory, and S. Diederichs, "Many roads to maturity: microRNA biogenesis pathways and their regulation," Nature Cell Biology, vol. 11, no. 3, pp. 228-234, 2009.

[23] S. Hara, M. Oya, R. Mizuno, A. Horiguchi, K. Marumo, and M. Murai, "Akt activation in renal cell carcinoma: contribution of a decreased PTEN expression and the induction of apoptosis by an Akt inhibitor," Annals of Oncology, vol. 16, no. 6, pp. 928-933, 2005.

[24] D. Koul, R. Shen, A. Garyali, L. Ke, T.-J. Liu, and W. Yung, "MMAC/PTEN tumor suppressor gene regulates vascular endothelial growth factor-mediated angiogenesis in prostate cancer," International Journal of Oncology, vol. 21, p. 469, 2002.

[25] J. H. F. Wixted, J. L. Rothstein, and L. C. Eisenlohr, "Identification of functionally distinct TRAF proinflammatory and phosphatidylinositol 3-kinase/mitogen-activated protein kinase/extracellular signal-regulated kinase kinase (PI3K/MEK) 
transforming activities emanating from RET/PTC fusion oncoprotein," Journal of Biological Chemistry, vol. 287, no. 6, pp. 3691-3703, 2012.

[26] S. Xie, M. Chen, B. Yan, X. He, X. Chen, and D. Li, "Identification of a role for the PI3K/AKT/mTOR signaling pathway in innate immune cells," PLoS One, vol. 9, no. 4, Article ID e94496, 2014.

[27] S. A. Melo and M. Esteller, "Dysregulation of microRNAs in cancer: playing with fire," FEBS Letters, vol. 585, no. 2011, pp. 2087-2099, 2011.

[28] L. Zhang, J. Huang, N. Yang et al., "microRNAs exhibit high frequency genomic alterations in human cancer," Proceedings of the National Academy of Sciences, vol. 103, no. 24, pp. 9136-9141, 2006.

[29] Y. Peng and C. M. Croce, "The role of microRNAs in human cancer," Signal Transduction and Targeted Therapy, vol. 1, no. 1, Article ID 15004, 2016.

[30] G. T. Iswariya, B. Paital, P. R. Padma, and R. Nirmaladevi, "microRNAs: epigenetic players in cancer and aging," Frontiers in Bioscience (Scholar Edition), vol. 11, pp. 29-55, 2019.

[31] W. Y. Ho and S. K. Yeap, "Phenotypic and mirna transcriptomic evaluation of cellular tumour spheroid as model of breast cancer stem cell studies," European Journal of Cancer, vol. 61, pp. S86-S87, 2016.

[32] E. O'Day and A. Lal, "MicroRNAs and their target gene networks in breast cancer," Breast Cancer Research, vol. 12, p. 201, 2010.

[33] S. Volinia, G. A. Calin, C.-G. Liu et al., "A microRNA expression signature of human solid tumors defines cancer gene targets," Proceedings of the National Academy of Sciences, vol. 103, no. 7, pp. 2257-2261, 2006.

[34] S. O’Bryan, S. Dong, J. M. Mathis, and S. K. Alahari, “The roles of oncogenic miRNAs and their therapeutic importance in breast cancer," European Journal of Cancer, vol. 72, pp. 1-11, 2017.

[35] F. Asghari, N. Haghnavaz, B. Baradaran, M. Hemmatzadeh, and T. Kazemi, "Tumor suppressor microRNAs: targeted molecules and signaling pathways in breast cancer," Biomedicine \& Pharmacotherapy, vol. 81, pp. 305-317, 2016.

[36] Y. Shimono, M. Zabala, R. W. Cho et al., "Downregulation of miRNA-200c links breast cancer stem cells with normal stem cells," Cell, vol. 138, no. 3, pp. 592-603, 2009.

[37] S. Vimalraj, P. J. Miranda, B. Ramyakrishna, and N. Selvamurugan, "Regulation of breast cancer and bone metastasis by microRNAs," Disease Markers, vol. 35, pp. 369-387, 2013.

[38] J. Tang, A. Ahmad, and F. H. Sarkar, "The role of microRNAs in breast cancer migration, invasion and metastasis," International Journal of Molecular Sciences, vol. 13, no. 12, pp. 13414-13437, 2012.

[39] P. J. Kaboli, A. Rahmat, P. Ismail, and K.-H. Ling, "Micrornabased therapy and breast cancer: a comprehensive review of novel therapeutic strategies from diagnosis to treatment," Pharmacological Research, vol. 97, pp. 104-121, 2015.

[40] M. V. Iorio, P. Casalini, C. Piovan, L. Braccioli, and E. Tagliabue, "Breast cancer and microRNAs: therapeutic impact," The Breast, vol. 20, no. 3, pp. S63-S70, 2011.

[41] H. Zhu, H. Wu, X. Liu et al., "Role of microrna mir-27a and mir-451 in the regulation of mdr1/p-glycoprotein expression in human cancer cells," Biochemical Pharmacology, vol. 76, no. 5, pp. 582-588, 2008.
[42] J. N. Goh, S. Y. Loo, A. Datta et al., "Micrornas in breast cancer: regulatory roles governing the hallmarks of cancer," Biological Reviews, vol. 91, no. 2, pp. 409-428, 2016.

[43] Z. Liang, H. Wu, J. Xia et al., "Involvement of mir-326 in chemotherapy resistance of breast cancer through modulating expression of multidrug resistance-associated protein 1," Biochemical Pharmacology, vol. 79, no. 6, pp. 817-824, 2010.

[44] N. R. Shah, "MicroRNAs in pathogenesis of breast cancer: implications in diagnosis and treatment," World Journal of Clinical Oncology, vol. 5, no. 2, p. 48, 2014.

[45] M. A. Chaudhry, "Radiation-induced microRNA: discovery, functional analysis, and cancer radiotherapy," Journal of Cellular Biochemistry, vol. 115, no. 3, pp. 436-449, 2014.

[46] E. L. Hayes and J. S. Lewis-Wambi, "Mechanisms of endocrine resistance in breast cancer: an overview of the proposed roles of noncoding RNA," Breast Cancer Research, vol. 17, no. 1, p. 40, 2015.

[47] P. Muluhngwi and C. M. Klinge, "Identification of miRNAs as biomarkers for acquired endocrine resistance in breast cancer," Molecular and Cellular Endocrinology, vol. 456, pp. 76-86, 2017. 